\title{
Spatial scales of the magnetic ramp at the Venusian bow shock
}

\author{
A. P. Dimmock ${ }^{1}$, S. N. Walker ${ }^{1}$, T. L. Zhang ${ }^{2}$, and S. A. Pope ${ }^{1}$ \\ ${ }^{1}$ Automatic Control \& Systems Engineering, University of Sheffield, Sheffield, UK \\ ${ }^{2}$ Space Research Institute of Austrian Academy of Sciences, Graz, Austria
}

Received: 19 August 2011 - Revised: 4 October 2011 - Accepted: 15 October 2011 - Published: 22 November 2011

\begin{abstract}
Typically multi-spacecraft missions are ideally suited to the study of shock spatial scales due to the separation of temporal and spatial variations. These missions are not possible at all locations and therefore in-situ multispacecraft measurements are not available beyond the Earth. The present paper presents a study of shock spatial scales using single spacecraft measurements made by the Venus Express spacecraft. The scales are determined based on previous knowledge of shock overshoot scales measured by the ISEE and Cluster missions. The study encompasses around 60 crossings of the Venusian bow shock from 2006 to 2009. The statistical relationship between the shock ramp spatial scales, overshoot and upstream shock parameters are investigated. We find that despite somewhat different solar wind conditions our results are comparable with those based on multi-spacecraft missions at the terrestrial bow shock.
\end{abstract}

Keywords. Interplanetary physics (Planetary bow shocks) Space plasma physics (Shock waves)

\section{Introduction}

Collision-less shocks are one of the core areas of plasma physics research. The dominant process that occurs at the front of a collision-less shock is the redistribution of the upstream plasma bulk kinetic energy into more degrees of freedom and the acceleration of a fragment of the particles into very high energies (Sagdeev, 1966; Sagdeev and Galeev, 1969). Instabilities within the shock front have also been attributed to the re-distribution of the upstream flow energy of which a comprehensive review was performed by Papadopoulos (1985). However, these energy distribution processes are also closely related to the spatial scales of the layers within the shock transition such as the foot, ramp

Correspondence to: A. P. Dimmock (a.dimmock@sheffield.ac.uk) and overshoot (supercritical shocks) regions. Studies have also established that the electron dynamics at the shock front (e.g. demagnetisation and thermalisation) are influenced by the shock spatial scales within the transition (Balikhin and Gedalin, 1994; Gedalin et al., 1995a,b; Balikhin et al., 1998). Typically the magnetic transition across the collision-less shock is the most commonly studied due to the reliability and availability of magnetic field measurements. As a result, the magnetic profile of the collision-less shock has been comprehensively studied in the space environment (Scudder et al., 1986; Farris et al., 1991; Krasnoselskikh et al., 1991; Newbury and Russell, 1996; Balikhin et al., 2002; Bale et al., 2005). Numerical simulations such as those by performed by Leroy et al. (1982) have also played a critical role in our knowledge of shock structure. As a consequence, there is currently a good understanding of shock structure however the roles that small scale structures have on individual dissipation mechanisms at the shock front still remain unclear.

Planetary bow shocks are the most commonly researched shocks. Naturally the Earth's bow shock has been the focus of the majority of studies due to the abundance of spacecraft (i.e. ISEE, ACE, THEMIS, Cluster) and the rich source of in-situ measurements made available over the decades. However in contrast, Venus has no significant intrinsic magnetic field (Phillips and Russell, 1987) and therefore the solar wind interaction is somewhat different interacting directly with the upper atmosphere (Luhmann, 1986). On the contrary, only a small amount of spacecraft (Pioneer Venus, Magellan, Venus Express) have been completely devoted to Venus study. The launch of Venus Express (VEX) allowed continual magnetic coverage of the surrounding Venus environment and lead to several publications regarding the Venusian bow shock (Balikhin et al., 2008; Zhang et al., 2008a,b; Whittaker et al., 2010). Currently no study has been dedicated to the spatial scales of the structures within the Venusian bow shock because typically single spacecraft missions such as VEX are not ideally appropriate for these types of shock studies.

Published by Copernicus Publications on behalf of the European Geosciences Union. 
Multi-spacecraft missions such as ISEE, THEMIS and Cluster are most suited to shock scale studies due to the ability to separate temporal and spatial variations within the shock structure but also the capability of the determination of accurate shock parameters. It is therefore unsurprising that no study of spatial scales relating the Venusian bow shock has been performed in comparison with the Earth terrestrial bow shock where several have been carried out (Livesey et al., 1982; Mellott and Livesey, 1987; Balikhin et al., 1995; Hobara et al., 2010). Recent missions such as Cluster have also permitted studies of electric field spatial scales within the shock transition such as the work performed by Walker et al. (2004); Dimmock et al. (2011), however this paper will concentrate solely on scales within the magnetic transition. In spite of the above restrictions, previous shock research has devised methods for the estimation of spatial scales using only single spacecraft measurements. Newbury et al. (1997) proposed a method in which the spatial scale of the shock ramp can be determined based on the non-coplanar component of the magnetic field at the shock front. However current availability of VEX MAG data is limited to $1 \mathrm{~Hz}$ time resolution therefore this method is not practical as is requires higher spatial detail across the shock ramp region. The spatial scales can also be estimated based on prior knowledge of the thickness of the foot and overshoot regions within the transition layer. A study by Livesey et al. (1982) showed that for the majority of shocks the overshoot spatial scale is of the order of three times the upstream ion gyro-radius this is also recently confirmed by Saxena et al. (2005) where the wavelength of the overshoot/undershoot was 2-3 ion gyro radii. The overshoot is defined as the region starting where the magnetic field first increases above the average upstream value and stops at the minimum point in the shock undershoot. A numerical measure of the overshoot is defined by Eq. (1)

$A=\frac{B_{\mathrm{o}}-B_{\mathrm{d}}}{B_{\mathrm{d}}}$

where $B_{0}$ is the maximum magnetic field value in the overshoot and $B_{\mathrm{d}}$ is the average downstream magnetic field value. This is obviously limited to shocks where the upstream Mach number exceeds that of the critical value as magnetic overshoot is a feature strictly of shocks only in the supercritical regime (Leroy et al., 1982). Nevertheless it allows for a reliable estimate of the overshoot width for single spacecraft missions such as Venus Express. As a result, it is possible to estimate other spatial scales within the shock transition such as the foot and ramp regions where only single datasets are available. The ramp in this paper is defined as the period in which the magnetic field undergoes the steepest gradient.

The purpose of this paper is to explore the spatial scale of the shock ramp and the dependance on the parameters of the quasi-perpendicular shock such as the upstream Alfvén Mach number $\left(M_{\mathrm{A}}\right)$ and the angle between the normal and the upstream magnetic field $\left(\Theta_{B \mathrm{n}}\right)$. The dependance of the overshoot will also be considered. Presented in this paper is a statistical study of around 60 crossings of the Venusian bow shock observed by VEX for a period from 2006 to 2009. The following section will discuss the datasets, instrumentation and technical implications, then two examples of shocks will be discussed to provide an accurate representation of the crossings observed during the course of this work. Finally the results will be considered together with the scientific and physical interpretations.

\section{Data and instrumentation}

All the shocks analysed for this study were observed by the Venus Express spacecraft over a period from 2006 to 2009. Of the wealth of shocks available over this period around 60 were analysed, the selection criteria leading to this will be outlined later. Magnetic field measurements used to identify the shock crossings were recorded by the Venus Express Magnetometer (Zhang et al., 2006) (VEX MAG) at a time resolution of $1 \mathrm{~Hz}$. Due to the absence of any magnetic cleanliness program for VEX, extensive cleaning was initially required of the VEX MAG datasets (Pope et al., 2011). Magnetic offset determination was also required (Leinweber et al., 2008) prior to the transformation into the Venusian coordinate system (VSO). The VSO system resembles the GSE system such that the $\mathrm{x}$-axis is directed Sunwards, the y direction opposes the spacecraft orbital motion, and the $\mathrm{z}$ axis is northward thus completing the right handed set. The ion temperature $\left(T_{\mathrm{i}}\right)$, density $\left(N_{\mathrm{p}}\right)$ and upstream solar wind velocity $\left(\boldsymbol{V}_{\mathrm{u}}\right)$ used to calculate the upstream ion gryoradius and Alfvén Mach number were all measured by the Analyser of Space Plasma and Energetic Atoms (ASPERA) instrument (Barabash et al., 2007).

\section{Shock crossings}

Around 60 shocks make up the statistical dataset in this study. Initially the magnetic profile of the shock transition determined shock selection. Following this, the selection criteria placed prime importance on the determination of an accurate shock normal $(\hat{\boldsymbol{n}})$. All normals were determined by minimum variance analysis (MVA) (Sonnerup and Cahill, 1967) and validated by the projection of the magnetic field directed along the shock normal. If sufficient upstream to downstream variation was seen, the normal was deemed unsuitable. Multiple periods across the shock were also applied to check for normal variability. In cases where normals varied by a large degree or no clear normal could be identified, the shock was not included. The quality status caveats of the ASPERA datasets were also taken into account where each data is marked between poor and excellent. Taking this in account, only satisfactory and above data were selected for this study. The shock spacecraft velocity $V_{\mathrm{Sh}}$ was calculated by dividing the spatial scale of the overshoot region by its time 


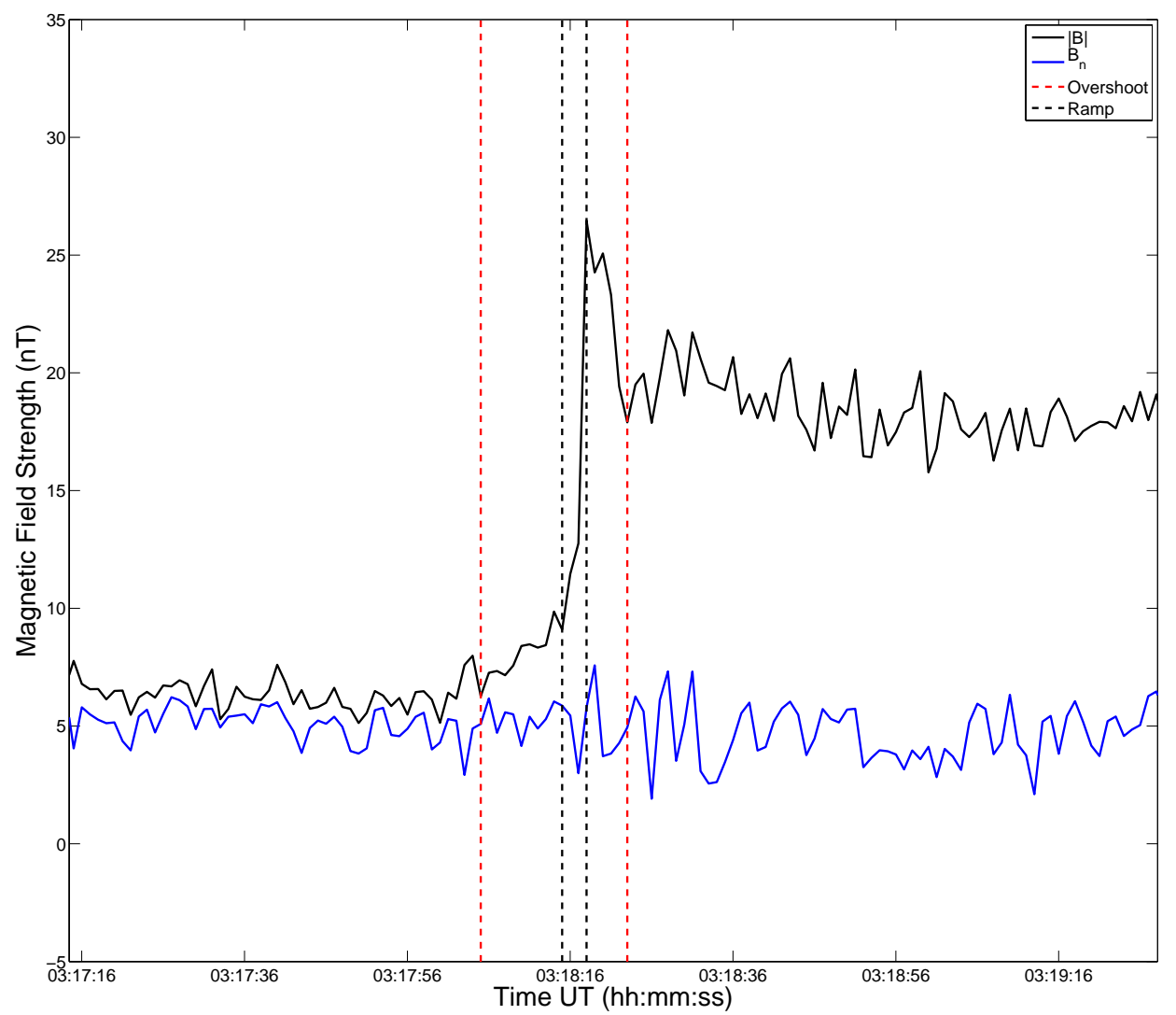

Fig. 1. A crossing of the Venusian bow shock on 24 April 2008. The black line shows the magnetic field profile measured by the VEX MAG instrument. The blue plot shows the magnetic field directed along the shock normal. Two red dashed lines show the interval of the magnetic overshoot used to determine the shock spatial scales.

duration which was used to determine the spatial scale of the magnetic ramp for each of the individual crossings.

Two example shocks will be discussed to demonstrate the analysis and assumptions that took place during this study. Both examples describe an accurate representation of the shocks encountered during this study of which are both included in the results.

\section{Shock 1: 24 April 2008}

Figure 1 displays a shock crossing observed by VEX on 24 April 2008 at 03:18:16 UT at an orbital radius of approximately $1.7 R_{\mathrm{v}}$. The black line shows the magnetic profile of the shock observed by the VEX MAG whereas the blue line shows the magnetic field directed along the shock normal. The overshoot and ramp for this shock are defined by the interval shown by the dashed vertical red and black lines, respectively. The shock profile exhibits typical behaviour of a supercritical quasi-perpendicular shock crossing evident by the clearly defined foot, ramp and overshoot features. Upstream of the shock the average magnetic field was measured as $\left|\boldsymbol{B}_{\mathrm{Up}}\right| \approx 6 \mathrm{nT}$ and the ASPERA instrument recorded a so- lar wind velocity of $\left|\boldsymbol{V}_{\mathrm{Up}}\right| \approx 360 \mathrm{~km} \mathrm{~s}^{-1}$. The shock normal was determined by MVA and was calculated as $[0.61,-0.75$, 0.24]. $B_{\mathrm{n}}$ exhibits little to no variation from upstream to downstream of the shock where fluctuations during the ramp region are also negligible with respect to the magnitude of the shock ramp. The shock geometry is quasi-perpendicular as the angle between $\hat{\boldsymbol{n}}$ and $\boldsymbol{B}_{\mathrm{Up}}$ was determined to be $\Theta_{B \mathrm{n}} \approx$ $49^{\circ}$. The upstream proton density was measured as $N_{\mathrm{p}} \approx$ $15 \mathrm{~cm}^{-3}$ resulting in an Alfvén Mach number of $M_{\mathrm{A}} \approx 4.7$ which confirms the supercritical nature of the shock consistent with the magnetic field profile in Fig. 1. The ion temperature measured upstream of the shock front was $T_{\mathrm{i}} \approx 5 \mathrm{eV}$ which was used to calculate the upstream ion gyro-radius $\left(R_{\mathrm{i}}=1.02 \times 10^{2} \sqrt{\mu} Z^{-1} \sqrt{T}_{\mathrm{i}}\left|\boldsymbol{B}_{\mathrm{Up}}\right|^{-1}=36.9 \mathrm{~km}\right.$, where $\mu$ and $Z$ are the ion/proton mass ratio and charge state, respectively) which indicated an overshoot spatial scale of $O_{\mathrm{Sc}} \approx$ $110.7 \mathrm{~km}$. The time duration of the overshoot $(A \approx 0.4)$ was calculated from the magnetic field data as $18 \mathrm{~s}$ which resulted in $V_{\mathrm{Sh}} \approx 6.15 \mathrm{~km} \mathrm{~s}^{-1}$. Based on the estimated $V_{\mathrm{Sh}}$, the spatial scale of the shock ramp was $R_{\mathrm{Sc}} \approx 8.7 \mathrm{~km}$ which is $0.3 R_{\mathrm{i}}$. 


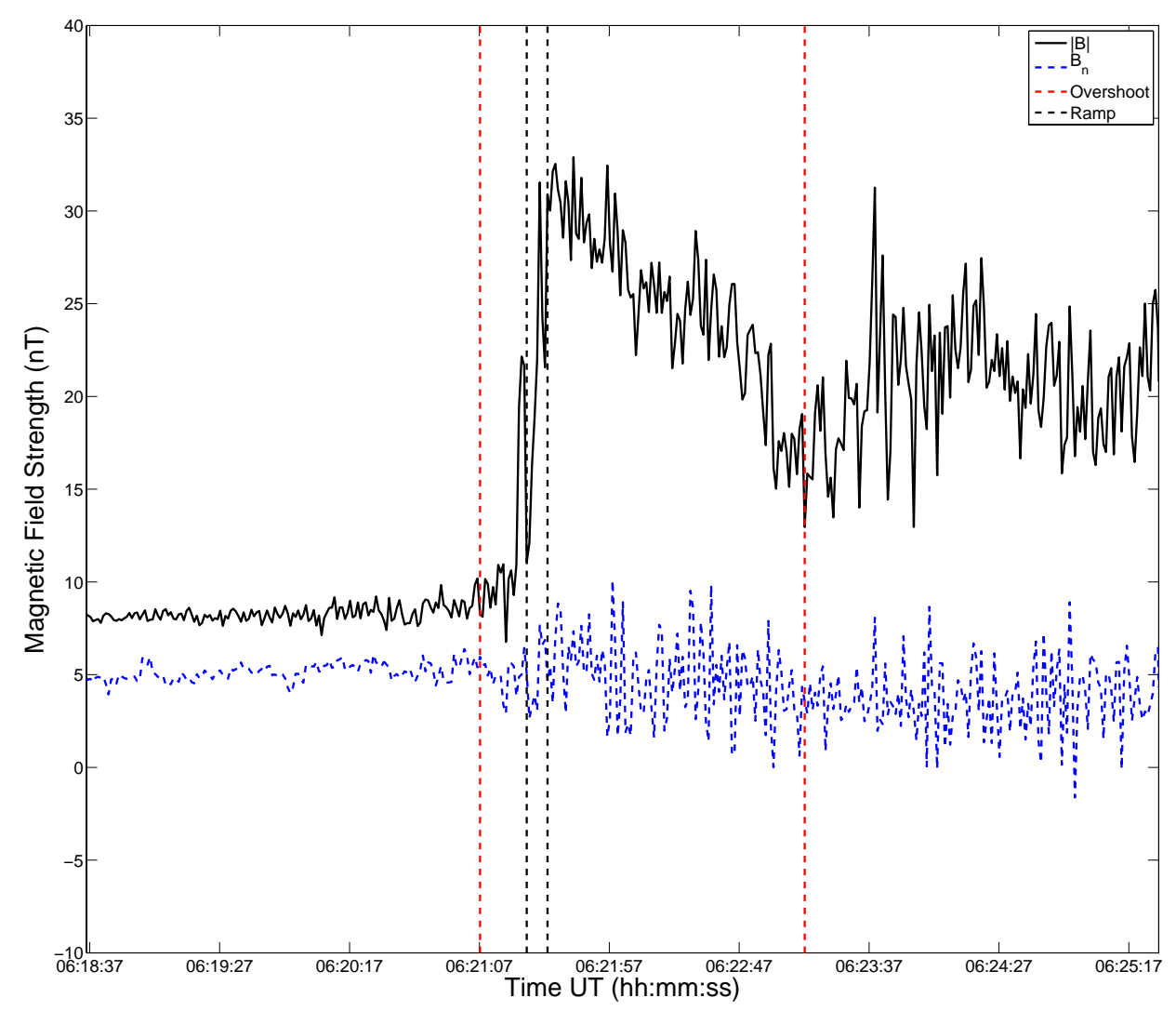

Fig. 2. A crossing of the Venusian bow shock on 11 June 2007. The black line shows the magnetic field profile measured by the VEX MAG instrument. The blue plot shows the magnetic field directed along the shock normal. Two red dashed lines show the interval of the magnetic overshoot used to determine the shock spatial scales.

\section{Shock 2: 11 June 2007}

Figure 2 represents a crossing of the Venusian bow shock that took place on 11 June 2007 at a distance of approximately $1.8 R_{\mathrm{v}}$. The black line represents the magnitude of the magnetic field profile whereas the blue line shows $B_{\mathrm{n}}$. The overshoot and ramp for this shock are defined by the interval shown by the dashed vertical red and black lines, respectively. The profile exhibits typical features which identify the shock as supercritical, notably the obvious overshoot following the ramp. The overshoot is arguably larger than the previous example suggesting a more elevated $M_{\mathrm{A}}$. Upstream measurements made by VEX indicated $\left|\boldsymbol{B}_{\mathrm{Up}}\right| \approx 7.6 \mathrm{nT}$ and $\left|\boldsymbol{V}_{\mathrm{Up}}\right| \approx 502 \mathrm{~km} \mathrm{~s}^{-1}$. The shock normal was determined using MVA and was $[0.67,0.73,0.15]$. The projection shows minimal upstream to downstream variation and change during the ramp. There is a small offset from upstream to far downstream however this correlates directly with the large overshoot, and due to a larger amount of low frequency plasma structures superimposed on the crossing there is sufficiently larger fluctuations with respect to the previous case. Nevertheless, the variation in $B_{\mathrm{n}}$ across the shock is negligible with respect to the change observed in $|\boldsymbol{B}|$ during the shock ramp. $\hat{n}$ resulted in $\Theta_{B \mathrm{n}} \approx 71^{\circ}$. Upstream measurements performed by the ASPERA instrument indicated $N_{\mathrm{p}}$ and $T_{\mathrm{i}}$ to be $4.5 \mathrm{~cm}^{-3}$ and $20 \mathrm{eV}$, respectively. $N_{\mathrm{p}}$ resulted in $M_{\mathrm{A}} \approx 5.5$ which supports the substantial overshoot observed in Fig. 2 which was calculated as 0.6. The ion gyro radius was determined to be $60 \mathrm{~km}$ which provides a spatial scale estimate of $O_{\mathrm{Sc}} \approx 180 \mathrm{~km}$. $V_{\mathrm{Sh}}$ was calculated as approximately $1.44 \mathrm{~km} \mathrm{~s}^{-1}$ which indicates the shock ramp spatial scale to be $11.26 \mathrm{~km}$ or $0.19 R_{\mathrm{i}}$.

\section{Results and discussion}

Figure 3 shows the estimated shock ramp spatial scales normalised with respect to the ion gyro-radius plotted against the Alfvén Mach number. The black crosses show the individual shocks whereas the red markers show the means between each intermediate Mach number. The red dashed line shows a tendency for the normalised ramp scale to decrease with increasing Alfvén Mach number. This statistical trend has also been observed by (Hobara et al., 2010) using Cluster measurements at the Earth bow shock. More notably the majority of shocks ramp scales tend to be $0.5 R_{\mathrm{i}}$ with lower and upper limits of 0.1 to $1 R_{\mathrm{i}}$, respectively. These limits are 


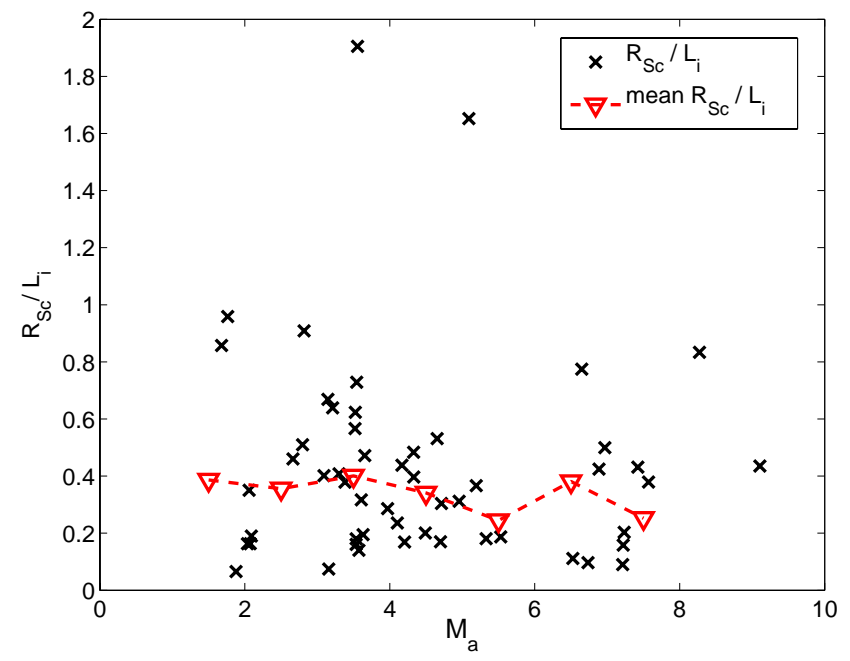

Fig. 3. The normalised shock ramp spatial scales plotted against Alfvén Mach number. The black markers show the individual crossings whereas the red dashed line shows the mean values for Mach numbers between (1-2, 3-4, 4-5 etc.). The means do not take into account the two outliers around 1.6 and 1.8.

in close agreement with shock ramp widths measured at the Earth using multi-spacecraft measurements by Cluster (Hobara et al., 2010), ISEE (Newbury et al., 1998) and AMPTE (Walker et al., 1999a) where typical $\left(1-3 R_{\mathrm{i}}\right)$ and very thin shock ramps have been observed.

There are two clear outliers in Fig. 3 however this could be explained by the fact that in some cases the assumption is incorrect although remains true for the majority of shock crossings. On the other hand there is no significant statistical dependency between $\Theta_{B \mathrm{n}}$ and the ramp width. The condition that $O_{\mathrm{Sc}} \approx 3 R_{\mathrm{i}}$ may not be entirely exact for all overshoots of supercritical shocks means that a case study would not be practical based on this assumption. However, statistically for the majority of shocks this should provide an accurate estimate of the ramp width and therefore will allow us to draw reliable conclusions.

Figure 4 shows the Alfvén Mach number plotted with the overshoot defined by Eq. (1). There is a strong tendency for the overshoot to increase with the Alfvén Mach number. Figure 5 shows the same data where in this case $\Theta_{B \mathrm{n}}$ is plotted with the overshoot. The statistical trend in Fig. 5 suggests that the distribution of the overshoot increases as $\Theta_{B \mathrm{n}} \rightarrow 90^{\circ}$ which is expected due to the increased number of ions returning back and accelerated towards the shock as geometry becomes perpendicular. It is also worth noting that there is some variation within the statistics, a major contribution to this could be the addition of turbulence within the shock transition in which the shock overshoot can be highly sensitive (Mellott and Livesey, 1987).

Overall the tendency for the shock ramp scale to decrease with the Alfvén Mach number validates the model that the whistler dispersion is the underlying process in the forma-

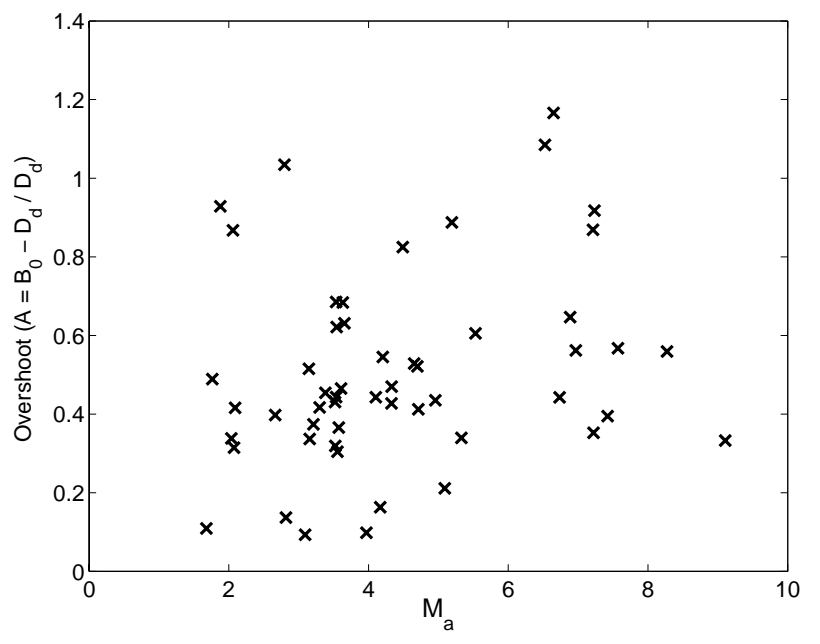

Fig. 4. Overshoot $\left(A=\frac{B_{0}-B_{\mathrm{d}}}{B_{\mathrm{d}}}\right)$ plotted against the Alfvén Mach number.

tion of the magnetic ramp. In the case of weak subcritical shocks, the stationary whistler precursor is formed as the result of the balance between the nonlinear steepening which transfers energy towards the short scales in the shock transition. The wave dispersion also allows waves with small enough wavelengths to leave the steepening region. The whistler critical Mach number $M_{\mathrm{w}}$ is the highest Mach number that still allows a linear whistler wave to phase stand in the upstream plasma flow (Kennel et al., 1985). In conditions where the upstream Mach number exceeds $M_{\mathrm{w}}$ a stationary wave precursor cannot be formed and the non stationary shock ramp is formed as the result of the counter balance between the nonlinearity and the dispersion of the high amplitude whistler waves (Krasnoselskikh, 1985; Balikhin et al., 1997a,b, 1999; Walker et al., 1999b). Another model of the magnetic ramp spatial scales of high Mach number and non stationary shocks are based on particle in cell (PIC) numerical simulations such as those by Lembege and Savoini (1992). According to this model, the small amplitude whistler grows at the upstream edge of the ramp to such high amplitudes that it replaces the shocks ramp and leads to shock reformation. In such a model the ramp scale should be spread in a significant range from a very short scales to scales that are compatible to the size of the foot region. The results presented in Fig. 3 do not support such a model. The absence of experimental support of this scenario can be explained by the artificially low ratio of the electron plasma frequency to the electron cyclotron frequency used in simulations. While for the terrestrial bow shock that ratio is typically $1.5 \times 10^{2}$, parameters of PIC simulations (Lembege and Savoini, 1992) result in a value of more than 1 order of magnitude lower. This leads to considerable overestimation of the electric to magnetic field ratio in the phase standing whistler waves which results in significant modification of their properties (Krasnoselskikh and Lobzin, 2009). 


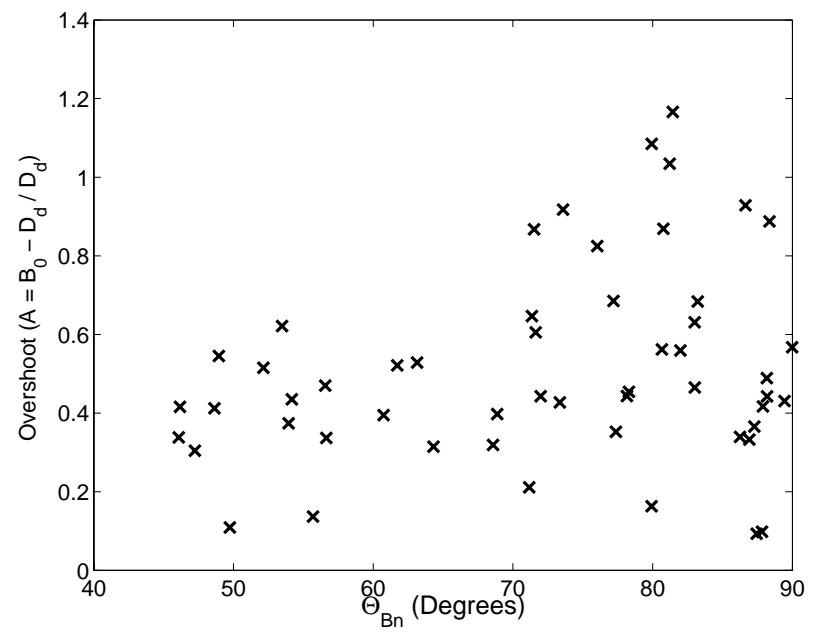

Fig. 5. Overshoot $\left(A=\frac{B_{\mathrm{o}}-B_{\mathrm{d}}}{B_{\mathrm{d}}}\right)$ plotted against $\Theta_{B \mathrm{n}}$.

\section{Conclusions}

The main conclusion that should be draw from this study is that despite the difference in solar wind conditions at the Venusian bow shock, and the absence of any significant intrinsic planetary magnetic field, the spatial scale of the ramp still lies within the same normalised values as studies carried out at the Earth bow shock. The spatial scales within the shock play a large role in the shock formation process and non-linearity at the shock front (Sagdeev, 1979; Papadopoulos, 1981; Kennel et al., 1985). The spatial scales within the shock transition also determine the types of mechanisms for the interaction between the upstream plasma flow and electromagnetic fields present at the shock front which also balance shock steepening. While there are many studies of spatial shock scales at the Earth (Livesey et al., 1982; Mellott and Livesey, 1987; Balikhin et al., 1995; Hobara et al., 2010) none have been performed based on the Venusian bow shock. The results of this study also demonstrate that the ability to study spatial scales at the shock front is not solely restricted to multi-spacecraft missions, and can allow for the study of shock spatial scales at locations where multi-spacecraft missions are unfeasible.

Acknowledgements. The authors would like to thank the VEX instrument teams for providing the datasets used in this study. The work was carried out under financial support from EPSRC and STFC.

Guest Editor M. Gedalin thanks two anonymous referees for their help in evaluating this paper.

\section{References}

Bale, S. D., Balikhin, M. A., Horbury, T. S., Krasnoselskikh, V. V., Kucharek, H., Möbius, E., Walker, S. N., Balogh, A., Burgess, D., Lembège, B., Lucek, E. A., Scholer, M., Schwartz, S. J., and Thomsen, M. F.: Quasi-perpendicular Shock Structure and Processes, Space Sci. Rev., 118, 161-203, doi:10.1007/s11214005-3827-0, 2005.

Balikhin, M. and Gedalin, M.: Kinematic mechanism of electron heating in shocks: Theory vs observations, Geophys. Res. Lett., 21, 841-844, doi:10.1029/94GL00371, 1994.

Balikhin, M., Krasnosselskikh, V., and Gedalin, M.: The scales in quasiperpendicular shocks, Adv. Space Res., 15, 247-260, doi:10.1016/0273-1177(94)00105-A, 1995.

Balikhin, M. A., de Wit, T. D., Alleyne, H. S. C. K., Woolliscroft, L. J. C., Walker, S. N., Krasnosel'skikh, V., MierJedrzejeowicz, W. A. C., and Baumjohann, W.: Experimental determination of the dispersion of waves observed upstream of a quasi-perpendicular shock, Geophys. Res. Lett., 24, 787-790, doi:10.1029/97GL00671, 1997a.

Balikhin, M. A., Walker, S. N., de Wit, T. D., Alleyne, H. S. C. K., Woolliscroft, L. J. C., Mier-Jedrzejowicz, W. A. C., and Baumjohann, W.: Non-stationarity and low frequency turbulence at a quasiperpendicular shock front, Adv. Space Res., 20, 729-734, doi:10.1016/S0273-1177(97)00463-8, 1997b.

Balikhin, M., Krasnoselskikh, V. V., Woolliscroft, L. J. C., and Gedalin, M.: A study of the dispersion of the electron distribution in the presence of $\mathrm{E}$ and $\mathrm{B}$ gradients: Application to electron heating at quasi-perpendicular shocks, J. Geophys. Res., 103, 2029-2040, doi:10.1029/97JA02463, 1998.

Balikhin, M. A., Alleyne, H., Treumann, R. A., Nozdrachev, M. N., Walker, S. N., and Baumjohann, W.: The role of nonlinear interaction in the formation of LF whistler turbulence upstream of a quasi-perpendicular shock, J. Geophys. Res. (Space Physics), 104, 12525-12536, doi:10.1029/1998JA900102, 1999.

Balikhin, M. A., Nozdrachev, M., Dunlop, M., Krasnoselskikh, V., Walker, S. N., Alleyne, H. S. C. K., Formisano, V., Andre, M., Balogh, A., Eriksson, A., and Yearby, K.: Observation of the terrestrial bow shock in quasi-electrostatic subshock regime, J. Geophys. Res. (Space Physics), 107, 1155, doi:10.1029/2001JA000327, 2002.

Balikhin, M. A., Zhang, T. L., Gedalin, M., Ganushkina, N. Y., and Pope, S. A.: Venus Express observes a new type of shock with pure kinematic relaxation, Geophys. Res. Lett., 35, L01103, doi:10.1029/2007GL032495, 2008.

Barabash, S., Sauvaud, J.-A., Gunell, H., Andersson, H., Grigoriev, A., Brinkfeldt, K., Holmström, M., Lundin, R., Yamauchi, M., Asamura, K., Baumjohann, W., Zhang, T. L., Coates, A. J., Linder, D. R., Kataria, D. O., Curtis, C. C., Hsieh, K. C., Sandel, B. R., Fedorov, A., Mazelle, C., Thocaven, J.-J., Grande, M., Koskinen, H. E. J., Kallio, E., Säles, T., Riihela, P., Kozyra, J., Krupp, N., Woch, J., Luhmann, J., McKenna-Lawlor, S., Orsini, S., Cerulli-Irelli, R., Mura, M., Milillo, M., Maggi, M., Roelof, E., Brandt, P., Russell, C. T., Szego, K., Winningham, J. D., Frahm, R. A., Scherrer, J., Sharber, J. R., Wurz, P., and Bochsler, P.: The Analyser of Space Plasmas and Energetic Atoms (ASPERA-4) for the Venus Express mission, Planet. Space Sci., 55, 1772-1792, doi:10.1016/j.pss.2007.01.014, 2007.

Dimmock, A. P., Balikhin, M. A., and Hobara, Y.: Comparison of three methods for the estimation of cross-shock electric potential using Cluster data, Ann. Geophys., 29, 815-822, doi:10.5194/angeo-29-815-2011, 2011.

Farris, M. H., Petrinec, S. M., and Russell, C. T.: The thickness of 
the magnetosheath - Constraints on the polytropic index, Geophys. Res. Lett., 18, 1821-1824, doi:10.1029/91GL02090, 1991.

Gedalin, M., Gedalin, K., Balikhin, M., and Krasnosselskikh, V.: Demagnetization of electrons in the electromagnetic field structure, typical for quasi-perpendicular collisionless shock front, J. Geophys. Res., 100, 9481-9488, doi:10.1029/94JA03369, 1995a.

Gedalin, M., Gedalin, K., Balikhin, M., Krasnosselskikh, V., and Woolliscroft, L. J. C.: Demagnetization of electrons in inhomogeneous $\mathrm{E} \perp \mathrm{B}$ : Implications for electron heating in shocks, J. Geophys. Res., 100, 19911-19918, doi:10.1029/95JA01399, 1995b.

Hobara, Y., Balikhin, M., Krasnoselskikh, V., Gedalin, M., and Yamagishi, H.: Statistical study of the quasi-perpendicular shock ramp widths, J. Geophys. Res. (Space Physics), 115, 11106, doi:10.1029/2010JA015659, 2010.

Kennel, C. F., Edmiston, J. P., and Hada, T.: A quarter century of collisionless shock research, Washington D.C. American Geophysical Union Geophysical Monograph Series, 34, 1-36, 1985.

Krasnoselskikh, V.: Nonlinear motions of a plasma across a magnetic field, Sov. Phys. Jetp, 62, 282-293, 1985.

Krasnoselskikh, V. and Lobzin, V.: Dynamical shock: Theory and observations, Eos Trans. AGU Fall meeting suppl., 90, SH42A02, 2009.

Krasnoselskikh, V. V., Vinogradova, T., Balikhin, M. A., Alleyne, H. S. C., Pardaens, A. K., Woolliscroft, L. J. C., Klimov, S. I., Petrukovich, A., Mier-Jedrzejowicz, W. A. C., and Southwood, D. J.: On the nature of low frequency turbulence in the foot of strong quasi-perpendicular shocks, Adv. Space Res., 11, 15-18, doi:10.1016/0273-1177(91)90002-2, 1991.

Leinweber, H. K., Russell, C. T., Torkar, K., Zhang, T. L., and Angelopoulos, V.: An advanced approach to finding magnetometer zero levels in the interplanetary magnetic field, Measurement Science and Technology, 19, 055 104, 2008.

Lembege, B. and Savoini, P.: Nonstationarity of a twodimensional quasiperpendicular supercritical collisionless shock by self-reformation, Phys. Fluids B, 4, 3533-3548, doi:10.1063/1.860361, 1992.

Leroy, M. M., Winske, D., Goodrich, C. C., Wu, C. S., and Papadopoulos, K.: The structure of perpendicular bow shocks, J. Geophys. Res., 87, 5081-5094, doi:10.1029/JA087iA07p05081, 1982.

Livesey, W. A., Kennel, C. F., and Russell, C. T.: ISEE-1 and 2 observations of magnetic field strength overshoots in quasiperpendicular bow shocks, Geophys. Res. Lett., 9, 1037-1040, doi:10.1029/GL009i009p01037, 1982.

Luhmann, J. G.: The solar wind interaction with Venus, Space Sci. Rev., 44, 241-306, doi:10.1007/BF00200818, 1986.

Mellott, M. M. and Livesey, W. A.: Shock overshoots revisited, J. Geophys. Res., 921, 13661-13665, doi:10.1029/JA092iA12p13661, 1987.

Newbury, J. A. and Russell, C. T.: Observations of a very thin collisionless shock, Geophys. Res. Lett., 23, 781-784, doi:10.1029/96GL00700, 1996.

Newbury, J. A., Russell, C. T., and Gedalin, M.: The determination of shock ramp width using the noncoplanar magnetic field component, Geophys. Res. Lett., 24, 1975-1978, doi:10.1029/97GL01977, 1997.

Newbury, J. A., Russell, C. T., and Gedalin, M.: The ramp widths of high-Mach-number, quasi-perpendicular collisionless shocks, J. Geophys. Res., 1032, 29581-29594, doi:10.1029/1998JA900024, 1998.

Papadopoulos, K.: Comments on high Mach number magnetosonic shocks, Tech. rep., European Space Agency, 1981.

Papadopoulos, K.: Microinstabilities and anomalous transport, Washington D.C. American Geophysical Union (Geophysical Monograph Series), 34, 59-90, 1985.

Phillips, J. L. and Russell, C. T.: Upper limit on the intrinsic magnetic field of Venus, J. Geophys. Res., 92, 2253-2263, doi:10.1029/JA092iA03p02253, 1987.

Pope, S. A., Zhang, T. L., Balikhin, M. A., Delva, M., Hvizdos, L., Kudela, K., and Dimmock, A. P.: Exploring planetary magnetic environments using magnetically unclean spacecraft: a systems approach to VEX MAG data analysis, Ann. Geophys., 29, 639647, doi:10.5194/angeo-29-639-2011, 2011.

Sagdeev, R. Z.: Cooperative Phenomena and Shock Waves in Collisionless Plasmas, Rev. Plasma Phys., 4, 23-90, 1966.

Sagdeev, R. Z.: The 1976 Oppenheimer lectures: Critical problems in plasma astrophysics. II. Singular layers and reconnection, Rev. Modern Physics, 51, 11-20, doi:10.1103/RevModPhys.51.11, 1979.

Sagdeev, R. Z. and Galeev, A. A.: Nonlinear Plasma Theory, W.A. Benjamin, 1969.

Saxena, R., Bale, S. D., and Horbury, T. S.: Wavelength and decay length of density overshoot structure in supercritical, collisionless bow shocks, Phys. Plasmas, 12, 052904 doi:10.1063/1.1900093, 2005.

Scudder, J. D., Aggson, T. L., Mangeney, A., Lacombe, C., and Harvey, C. C.: The resolved layer of a collisionless, high beta, supercritical, quasi-perpendicular shock wave. I - RankineHugoniot geometry, currents, and stationarity, J. Geophys. Res., 91, 11019-11052, doi:10.1029/JA091iA10p11019, 1986.

Sonnerup, B. U. O. and Cahill Jr., L. J.: Magnetopause Structure and Attitude from Explorer 12 Observations, J. Geophys. Res., 72, 171, doi:10.1029/JZ072i001p00171, 1967.

Walker, S. N., Balikhin, M. A., Alleyne, H. S. C. K., Baumjohann, W., and Dunlop, M.: Observations of a very thin shock, Adv. Space Res., 24, 47-50, doi:10.1016/S0273-1177(99)004214, 1999a.

Walker, S. N., Balikhin, M. A., and Nozdrachev, M. N.: Ramp nonstationarity and the generation of whistler waves upstream of a strong quasiperpendicular shock, Geophys. Res. Lett., 26, 13571360, doi:10.1029/1999GL900210, 1999b.

Walker, S. N., Alleyne, H. St. C. K., Balikhin, M. A., André, M., and Horbury, T. S.: Electric field scales at quasi-perpendicular shocks, Ann. Geophys., 22, 2291-2300, doi:10.5194/angeo-222291-2004, 2004.

Whittaker, I., Guymer, G., Grande, M., Pintér, B., Barabash, S., Federov, A., Mazelle, C., Sauvaud, J. A., Lundin, R., Russell, C. T., Futaana, Y., Fränz, M., Zhang, T. L., Andersson, H., Grigoriev, A., Holmström, M., Yamauchi, M., Asamura, K., Baumjohann, W., Lammer, H., Coates, A. J., Kataria, D. O., Linder, D. R., Curtis, C. C., Hsieh, K. C., Koskinen, H. E. J., Kallio, E., Riihelä, P., Schmidt, W., Kozyra, J., McKenna-Lawlor, S., Thocaven, J. J., Orsini, S., Cerulli-Irelli, R., Mura, A., Milillo, M., Maggi, M., Roelof, E., Brandt, P., Frahm, R. A., Sharber, J. R., Wurz, P., and Bochsler, P.: Venusian bow shock as seen by the ASPERA-4 ion instrument on Venus Express, J. Geophys. 
Res. (Space Physics), 115, A09224, doi:10.1029/2009JA014826, 2010.

Zhang, T. L., Baumjohann, W., Delva, M., Auster, H.-U., Balogh, A., Russell, C. T., Barabash, S., Balikhin, M., Berghofer, G., Biernat, H. K., Lammer, H., Lichtenegger, H., Magnes, W., Nakamura, R., Penz, T., Schwingenschuh, K., Vörös, Z., Zambelli, W., Fornacon, K.-H., Glassmeier, K.-H., Richter, I., Carr, C., Kudela, K., Shi, J. K., Zhao, H., Motschmann, U., and Lebreton, J.-P.: Magnetic field investigation of the Venus plasma environment: Expected new results from Venus Express, Planet. Space Sci., 54, 1336-1343, doi:10.1016/j.pss.2006.04.018, 2006.
Zhang, T. L., Delva, M., Baumjohann, W., Volwerk, M., Russell, C. T., Barabash, S., Balikhin, M., Pope, S., Glassmeier, K.H., Kudela, K., Wang, C., Vörös, Z., and Zambelli, W.: Initial Venus Express magnetic field observations of the Venus bow shock location at solar minimum, Planet. Space Sci., 56, 785789, doi:10.1016/j.pss.2007.09.012, 2008a.

Zhang, T. L., Pope, S., Balikhin, M., Russell, C. T., Jian, L. K., Volwerk, M., Delva, M., Baumjohann, W., Wang, C., Cao, J. B., Gedalin, M., Glassmeier, K.-H., and Kudela, K.: Venus Express observations of an atypically distant bow shock during the passage of an interplanetary coronal mass ejection, J. Geophys. Res. (Planets), 113, E00B12, doi:10.1029/2008JE003128, 2008 b. 